\title{
Modified aortoplasty for discrete congenital supravalvular aortic stenosis
}

\author{
Matthew L. Stone, MD, Ahmet Kilic, MD, Irving L. Kron, MD, and James J. Gangemi, MD, \\ Charlottesville, Va
}

Congenital supravalvular aortic stenosis (SVAS) is a rare obstructive lesion of the left ventricular outflow tract, and novel surgical treatments have fostered improved outcomes and survival for children with this condition. We present here a successful 3-sinus patch reconstruction for SVAS with extended patch augmentation to the right coronary sinus of Valsalva, maintaining aortic root geometry and providing a simplified approach for ascending aortic augmentation at the right coronary sinus.

\section{CASE REPORT}

A 5-year-old boy was seen at our institution with severe SVAS and the presumptive diagnosis of Williams-Beuren syndrome. His medical history was significant for birth at 34 weeks of gestational age, persistent developmental delay, reactive airway disease, and significant hearing loss. Pertinent physical features included dysmorphic facial features, microcephaly, and short stature. An echocardiogram obtained when he was an infant demonstrated mildly elevated aortic arch velocities with no focal evidence of stenosis or obstruction. He received developmental care and symptomatic surveillance at another institution and returned for cardiovascular consultation at 5 years of age because of progressive failure to thrive and the presence of a systolic ejection murmur. Echocardiographic findings established the presence of severe SVAS with a peak systolic gradient of $80 \mathrm{~mm} \mathrm{Hg}$ and a mean gradient of $52 \mathrm{~mm} \mathrm{Hg}$ with mild right pulmonary artery stenosis. Cardiac catheterization confirmed the diagnosis of severe SVAS with a peak systolic gradient of $90 \mathrm{~mm} \mathrm{Hg}$, a left ventricular enddiastolic pressure of $17 \mathrm{~mm} \mathrm{Hg}$, normal biventricular function, and moderate concentric left ventricular hypertrophy (Figure 1).

The family was counseled regarding the indications for operative intervention, and the patient was taken to the operating room at 5 years of age. A single aortic and bicaval cannula approach was used for the maintenance of

From the Division of Thoracic and Cardiovascular Surgery, Department of Surgery, University of Virginia Health System, Charlottesville, Va.

Disclosures: Authors have nothing to disclose with regard to commercial support.

Received for publication July 26, 2011; revisions received Oct 28, 2011; accepted for publication Dec 8, 2011; available ahead of print Jan 13, 2012.

Address for reprints: James J. Gangemi, MD, Division of Thoracic and Cardiovascular Surgery, University of Virginia Health System, PO Box 800679, Charlottesville, VA 22908 (E-mail: jgangemi@ virginia.edu).

J Thorac Cardiovasc Surg 2012;143:1450-1

$0022-5223 / \$ 36.00$

Copyright (c) 2012 by The American Association for Thoracic Surgery doi: $10.1016 /$ j.jtcvs.2011.12.011 cardiopulmonary bypass. The aorta was crossclamped with subsequent antegrade cardioplegia. The aorta was transected at the supravalvular site of stenosis, and incisions were made down into each coronary sinus. A straight-down incision was made into the noncoronary cusp. Incisions were then performed to the left of the right coronary artery and to the right of the left coronary artery within the respective aortic cusps. A triangular bovine pericardial patch was applied to augment the noncoronary and left coronary sinuses. The distal and proximal aortic segments were reapproximated posteriorly with a running 5-0 Prolene (Ethicon, Inc, Somerville, NJ) suture. The anterior portion of the aorta was repaired with an extended bovine pericardial patch augmentation and 5-0 Prolene suture (Figure 2). The patient was successfully weaned from cardiopulmonary bypass and transferred to the pediatric intensive care unit. Echocardiography on postoperative day three demonstrated a 14-mm $\mathrm{Hg}$ aortic gradient consistent with no residual SVAS. The patient was discharged home on day 4 of hospitalization with an uncomplicated postoperative course.

\section{DISCUSSION}

Congenital SVAS is the least common obstructive lesion of the left ventricular outflow tract. ${ }^{1}$ This malformation results in an obstructive arteriopathy that may manifest as a narrowing of the ascending aorta and arch branches or, less commonly, a discrete stenosis at the sinotubular junction as presented. ${ }^{1}$ Williams-Beuren syndrome is a disorder comprised of SVAS, hypercalcemia, and a spectrum of phenotypic variability that includes a distinctive facial appearance, mental retardation, and impaired growth. ${ }^{2}$ In addition to Williams-Beuren syndrome, SVAS may occur in isolation or as the result of autosomal dominant familial inheritance. ${ }^{1}$ This structural defect may impact pulmonary artery diameter, coronary artery flow, and aortic valve function. Preoperative catheterization provides an understanding of these potential concomitant anomalies, supporting an informed approach to reconstruction.

Novel surgical treatments for SVAS have fostered improved outcomes and survival for children with SVAS. Multiple-sinus reconstruction has established superiority relative to single-sinus augmentation, offering superior hemodynamics, decreased reoperations, and lower mortality. ${ }^{3}$ The 3-sinus approach, introduced by Brom, ${ }^{4}$ provides effective circumferential augmentation of the focal area of 


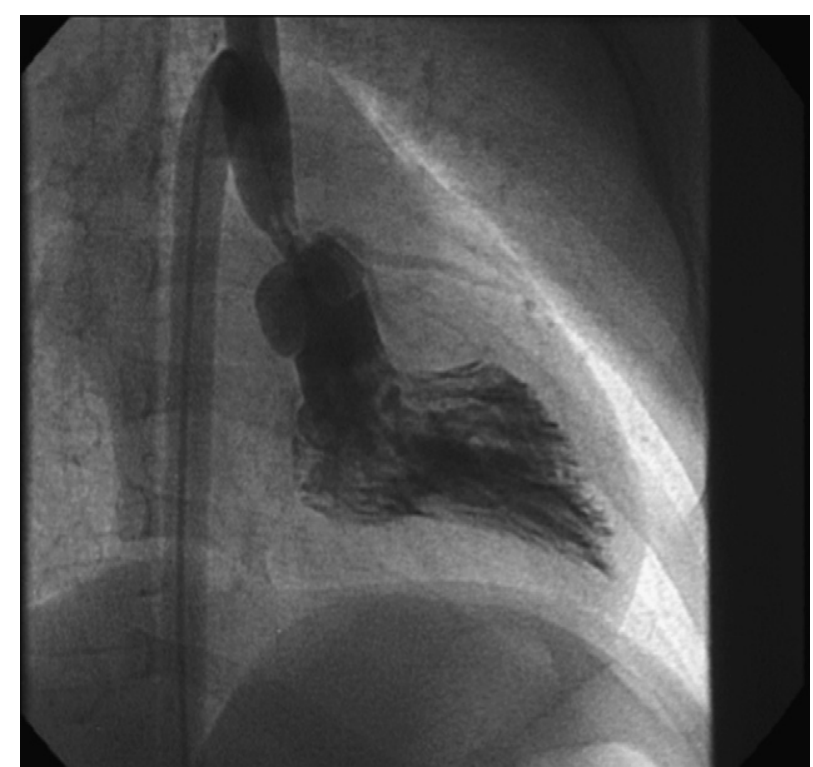

FIGURE 1. Preoperative cardiac catheterization image demonstrating severe supravalvular aortic stenosis.

SVAS. This repair may, however, provide inadequate longterm protection against restenosis at the new sinotubular junction. ${ }^{3}$ The foundational concept of ascending arch augmentation is addressed in the inverted 2-sinus Y-patch described by Doty and colleagues. ${ }^{5}$ This repair offers long-term protection against ascending arch restenosis while introducing the potential for untreated thickening at the left coronary sinus. ${ }^{1}$ An understanding of proven benefits and potential limitations to current methods for reconstruction supports the appropriate selection and design of individualized surgical treatment.

We present here a successful 3-sinus patch reconstruction for SVAS that applies an extended patch augmentation to the right coronary sinus of Valsalva. This repair maintains aortic root geometry while providing a simplified approach for ascending aortic augmentation at the right coronary sinus. The longitudinal incision along the distal aorta addresses the concern for restenosis potential inherent to the Brom repair. The right coronary sinus provides an anatomically accessible site for augmentation that limits aortic

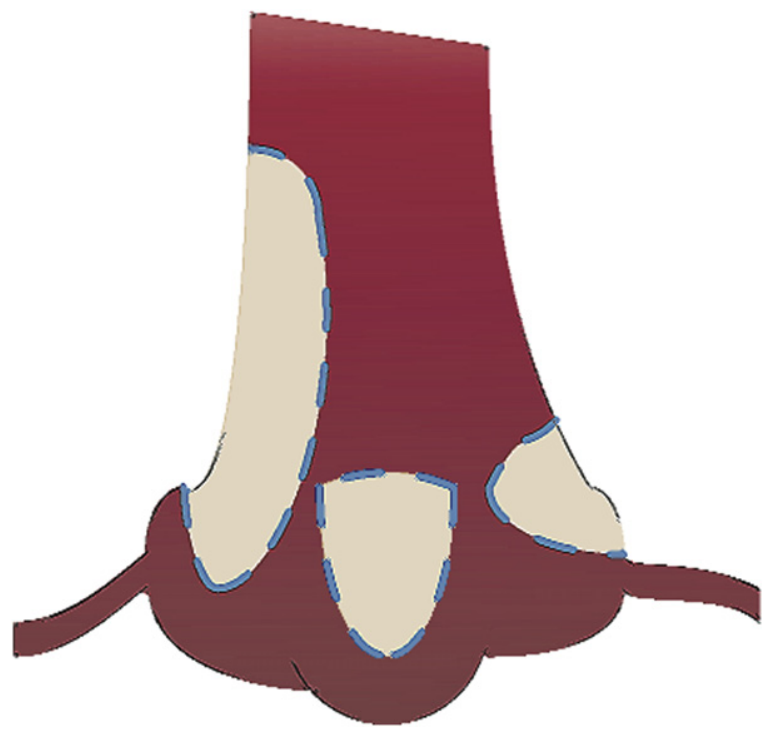

FIGURE 2. Depiction of repair with extended bovine pericardial patch augmentation of the right coronary sinus with concomitant triangular augmentation of the left coronary and noncoronary sinuses.

cross-clamp and cardiopulmonary bypass times. The application of this modification to the accepted benefits of multisinus repair supports an efficient and effective method for reconstruction. Although long-term outcomes remain unproven, we recommend consideration of this technique for children with SVAS limited to the aortic sinotubular junction.

\section{References}

1. Stamm C, Friehs I, Ho SY, Moran AM, Jonas RA, del Nido PJ. Congenital supravalvar aortic stenosis: a simple lesion? Eur J Cardiothorac Surg. 2001;19: 195-202.

2. Garcia RE, Friedman WF, Kaback MM, Rowe RD. Idiopathic hypercalcemia and supravalvular aortic stenosis. Documentation of a new syndrome. $N$ Engl J Med. 1964;271:117-20.

3. Stamm C, Kreutzer C, Zurakowski D, Nollert G, Friehs I, Mayer JE, et al. Fortyone years of surgical experience with congenital supravalvular aortic stenosis. J Thorac Cardiovasc Surg. 1999;118:874-85.

4. Brom A. Obstruction of the left ventricular outflow tract. In: Khonsari S, ed. Cardiac surgery: safeguards and pitfalls in operative technique. Rockville (MD): Lippincott Williams \& Wilkins; 1988. p. 276-80.

5. Doty DB, Polansky DB, Jensen CB. Supravalvular aortic stenosis: repair by extended aortoplasty. J Thorac Cardiovasc Surg. 1977;74:362-71. 